\title{
Anti-Citrullinated Cyclic Peptide Antibody and Functional Disability are Associated With Poor Sleep Quality in Rheumatoid Arthritis
}

\author{
Shamala RAJALINGAM, ${ }^{1}$ Rajalingham SAKTHISWARY, ${ }^{2}$ Heselynn HUSSEIN ${ }^{1}$ \\ ${ }^{1}$ Department of Medicine, Putrajaya Hospital, Putrajaya, Malaysia \\ ${ }^{2}$ Department of Medicine, University Kebangsaan Malaysia, Kuala Lumpur, Malaysia
}

\begin{abstract}
Objectives: This study aims to determine the predictors of poor sleep quality in rheumatoid arthritis (RA).

Patients and methods: This was a monocentric, cross sectional, case-control study which was conducted at the Putrajaya Hospital, Malaysia. We recruited 46 patients with RA ( 3 males; 43 females; mean age $48.15 \pm 14.96$ ) and 46 age and sex-matched healthy controls ( 3 males; 43 females; mean age $47.11 \pm 12.22$ ). RA patients were assessed for their disease activity based on disease activity score in 28 joints, disease damage based on radiographic erosions, and functional status based on Health Assessment Questionnaire Disability Index. The Pittsburgh Sleep Quality Index (PSQI) scores were determined by interviewing all the subjects. Subjects with RA were further subdivided based on their PSQI scores as "good sleepers" with PSQI scores of $<5$ and "poor sleepers" with PSQI scores of $\geq 5$.

Results: The percentage of poor sleepers was significantly higher among RA patients (47.83\% vs $9.57 \%$ ). Median scores of 5 out of 7 components of the PSQI were higher among RA patients compared to controls. Among poor sleepers with RA, a significantly higher proportion tested positive for anti-citrullinated cyclic peptide autoantibodies ( $p=0.037$ ). Besides, poor sleepers had significantly higher median Health Assessment Questionnaire Disability Index $(p=0.017)$ than good sleepers. However, both Health Assessment Questionnaire Disability Index ( $p=0.968)$ and anti-citrullinated cyclic peptide $(p=0.431)$ were insignificant when entered in the equation of a logistic regression model.

Conclusion: The findings of this study demonstrate a link between functional disability, anti-citrullinated cyclic peptide antibodies, and sleep quality in RA.

Keywords: Anti-citrullinated cyclic peptide; functional capacity; rheumatoid arthritis; sleep quality.
\end{abstract}

Rheumatoid arthritis (RA) is a chronic autoimmune disorder characterized by joint inflammation, joint destruction, and physical disability. ${ }^{1,2}$ Several studies have pointed out that 54 to $70 \%$ of RA patients report problems related to sleep, including difficulty falling asleep, poor sleep quality, nonrestorative sleep, wakefulness, awakenings during the night, and excessive daytime sleepiness. ${ }^{3,4}$

Sleep exerts a significant regulatory influence on immune functions through complex mechanisms which are poorly understood. Studies on the normal sleep-wake cycle revealed that immune parameters such as undifferentiated $\mathrm{T}$ cells and pro-inflammatory cytokines tend to peak during early nocturnal sleep whereas cytotoxic natural killer cells, as well as anti-inflammatory cytokines peak during daytime wakefulness. ${ }^{5,6}$ Comparisons of the effects of nocturnal sleep with sleep deprived subjects (24-hour periods of wakefulness) suggest that sleep facilitates the extravasation of $\mathrm{T}$ cells and their possible redistribution to lymph nodes. There seems to be a selectively enhancing influence of sleep on the interaction between antigen presenting cells and T helper cells. ${ }^{7}$

Research in neuroimmunology has accumulated substantial evidence indicating that sleep enhances immune defense, along the lines of lay people's

Received: February 11, 2016 Accepted: May 16, 2016 Published online: December 08, 2016

Correspondence: Rajalingham Sakthiswary, MD. Department of Medicine, Universiti Kebangsaan Malaysia, 43600 Kuala Lumpur, Malaysia.

Tel: 0391456099 e-mail: sakthis5@hotmail.com

○2017 Turkish League Against Rheumatism. All rights reserved. 
popular wisdom, "sleep helps healing". 8,9 Sleep reduces the production of interferon-gamma as well as tumor necrosis factor-alpha by cytotoxic T lymphocytes, ${ }^{10}$ which are key orchestrators in the pathogenesis of RA.

Sleep quality is an important component of the quality of life which is often overlooked by clinicians. Restorative sleep has an important role in maintaining health with disrupted or lower levels of sleep being related to an increased risk of co-morbidity and all-cause mortality. ${ }^{11}$ Thus, addressing poor sleep quality may be important in promoting health and well-being in patients with RA. In fact, recent clinical trials in RA have incorporated sleep quality as one of the outcome measures. ${ }^{12}$ Hence, in this study, we aimed to determine the predictors of poor sleep quality in RA.

\section{PATIENTS AND METHODS}

This was a monocentric, cross sectional, casecontrol study which was conducted between January 2015 and August 2015 at the Putrajaya Hospital, Malaysia. The study included 46 patients with RA (3 males, 43 females; mean age $48.15 \pm 14.96)$ and 46 age and sexmatched healthy controls ( 3 males, 43 females; mean age 47.11 \pm 12.22 ). A written informed consent was obtained from each patient. The study proposal was reviewed and approved by the Ethics \& Research Committee of the center (Study Code 21032). The study was conducted in accordance with the principles of the Declaration of Helsinki.

We recruited patients with RA from the rheumatology outpatient clinics. We invited patients who fulfilled the inclusion and exclusion criteria to participate in this study. We briefed individuals with poor command of English with the assistance of a translator.

The following were the inclusion criteria for RA patients: (i) fulfilling The American College of Rheumatology 2010 criteria for RA; $;^{13}$ (ii) being 18 years of age and above; (iii) being able to provide written informed consent. The following were the exclusion criteria: (i) being pregnant; (ii) having psychiatric disorders; (iii) having obstructive sleep apnea. We also applied the aforementioned exclusion criteria to the controls. Most of the controls in this study were health-care workers.

We recorded the demographic data such as age, sex, and race of all subjects. We collected data on disease duration and laboratory parameters by reviewing the computerized medical records of the RA patients. We assessed the patients for their disease activity based on disease activity score in 28 joints (DAS28) and performed hand and feet radiographs to examine any joint erosions. We used The Health Assessment Questionnaire Disability Index (HAQ-DI) for the evaluation of the functional status of the RA patients. We determined The Pittsburgh Sleep Quality Index (PSQI) scores by interviewing all the subjects. A single interviewer conducted the interviews to minimize bias.

Disease Activity Score in 28 joints is a quantitative measure of disease activity in RA. It has a validated formula which is used worldwide particularly in clinical trials. ${ }^{14}$ The four parameters used to calculate DAS28 are number of swollen joints, number of tender joints, C-reactive protein or erythrocyte sedimentation rate, and the patient's global assessment. ${ }^{15}$ DAS-28 score of 3.2 and above indicate moderate to severe disease activity whereas a value below 3.2 refers to remission to low disease activity. ${ }^{16}$

The HAQ-DI is the most widely used tool for functional assessment in RA. ${ }^{17}$ The HAQ-DI evaluates patients' level of functional ability involving the upper and lower limbs. There are 20 questions in eight categories which represent a comprehensive set of common daily activities: dressing, rising, eating, walking, and hygiene. Higher scores indicate more disability $(0=$ without any difficulty; $1=$ with some difficulty; $2=$ with much difficulty; and $3=$ unable to do). A HAQ-DI score of 1 and above indicate significant (moderate to very severe) functional disability. ${ }^{18}$

The PSQI measures patients' reported sleep quality over a period of one month. The PSQI comprises seven main components: (i) subjective sleep quality, (ii) sleep latency, (iii) sleep duration, (iv) habitual sleep efficiency, (v) sleep disturbances, (vi) use of sleep medications, and (vii) daytime dysfunction. In all seven components, a score of 0 indicates no difficulty whereas a score of 3 indicates severe difficulty. A PSQI 
score of 5 or above is recommended as the cutoff for sleep impairment. ${ }^{19}$

\section{Statistical analysis}

All data were analyzed using IBM SPSS version 21.0 software (IBM Corporation, Armonk, NY, USA). The continuous variables were tested for normality using Kolmogorov Smirnov test. Data with normal distribution were analyzed using independent t-test and expressed as mean \pm standard deviation. The data which were skewed were analyzed using Mann-Whitney U test and expressed as median \pm range. Variables which were significant on univariate analysis were further analyzed using multivariate analysis to adjust for confounders. The relationship between two continuous variables were determined using bivariate correlation analysis. A $p$ value of less than 0.05 was considered statistically significant.

\section{RESULTS}

As RA is more common among females, majority of the subjects $(93.48 \%)$ were females. The subjects were of three main ethnicities: Malays, Chinese, and Indians. The sociodemographic characteristics of RA patients and controls were matched (Table 1).

The percentage of poor sleepers (PSQI $\geq 5$ ) was significantly higher among RA patients $(47.83 \%$ vs 9.57\%). Median scores of 5 out of 7 components of the PSQI were higher among RA patients compared to controls. Compared to controls, RA patients had significantly higher median scores for sleep quality, sleep latency, sleep efficiency, and use of sleep medications. Global PSQI score was, therefore, significantly higher among RA patients $(p<0.05)$.

Table 2 compares the clinical characteristics of good sleepers and poor sleepers with RA.

Table 1. Demographic and clinical parameters of rheumatoid arthritis patients and controls

\begin{tabular}{|c|c|c|c|c|c|c|c|c|c|c|c|}
\hline \multirow[b]{2}{*}{ Parameter } & \multicolumn{5}{|c|}{ Rheumatoid arthritis patients $(n=46)$} & \multicolumn{5}{|c|}{ Controls $(n=46)$} & \multirow[b]{2}{*}{$p$} \\
\hline & $\mathrm{n}$ & $\%$ & Mean \pm SD & Median & Range & $\mathrm{n}$ & $\%$ & Mean \pm SD & Median & Range & \\
\hline Age (years) & & & $48.2 \pm 15.0$ & & & & & $47.1 \pm 12.2$ & & & 0.715 \\
\hline \multicolumn{12}{|l|}{ Sex } \\
\hline Female & 43 & 93.48 & & & & 43 & 93.48 & & & & \multirow{2}{*}{1.000} \\
\hline Male & 3 & 6.52 & & & & 3 & 6.52 & & & & \\
\hline Disease duration (years) & & & $9.2 \pm 5.6$ & & & & & NA & & & \\
\hline Seropositive & 33 & 71.74 & & & & & & NA & & & \\
\hline Anti CCP positive & 37 & 80.43 & & & & & & & & & \\
\hline Erosive disease & 19 & 41.30 & & & & & & NA & & & \\
\hline Pulmonary fibrosis & 12 & 26.10 & & & & & & NA & & & \\
\hline DAS28 & & & $3.0 \pm 1.5$ & & & & & NA & & & \\
\hline HAQ-DI & & & $0.8 \pm 0.8$ & & & & & NA & & & \\
\hline Poor sleeper & 22 & 47.83 & & & & 9 & 9.57 & & & & 0.008 \\
\hline Sleep quality score & & & & 1.00 & $0-3$ & & & & 0 & $0-2$ & 0.011 \\
\hline Sleep latency score & & & & 1.00 & $0-3$ & & & & 0 & $0-2$ & 0.024 \\
\hline Sleep duration score & & & & 0 & $0-3$ & & & & 1.00 & $0-2$ & 0.283 \\
\hline Sleep efficiency score & & & & 1.00 & $0-3$ & & & & 0 & $0-1$ & $<0.05$ \\
\hline Sleep disturbances score & & & & 1.00 & $0-3$ & & & & 0 & $0-3$ & 0.520 \\
\hline Sleep medication score & & & & 1.00 & $0-3$ & & & & 0 & $0-1$ & $<0.05$ \\
\hline $\begin{array}{l}\text { Daytime dysfunction } \\
\text { score }\end{array}$ & & & & 0 & $0-2$ & & & & 0 & $0-1$ & 0.116 \\
\hline Global PSQI & & & & 4.50 & $0-15$ & & & & 2.00 & $0-9$ & $<0.05$ \\
\hline
\end{tabular}


Table 2. Comparison between good sleepers and poor sleepers in rheumatoid arthritis

\begin{tabular}{|c|c|c|c|c|c|c|c|c|c|}
\hline \multirow[t]{2}{*}{ Parameter } & \multicolumn{4}{|c|}{ Good sleepers $(n=24)$} & \multicolumn{4}{|c|}{ Poor sleepers $(n=22)$} & \multirow[b]{2}{*}{$p$} \\
\hline & $\mathrm{n}$ & $\%$ & Median & Range & $\mathrm{n}$ & $\%$ & Median & Range & \\
\hline Age (years) & & & 46.50 & $21.00-80.00$ & & & 54.50 & $19.00-78.00$ & 0.353 \\
\hline Disease duration (years) & & & 8.00 & $1.00-22.00$ & & & 11.50 & $1.00-22.00$ & 0.234 \\
\hline Seropositive & 17 & 70.34 & & & 16 & 72.73 & & & 0.887 \\
\hline Anti-CCP positive & 16 & 66.67 & & & 21 & 95.45 & & & 0.037 \\
\hline Erosive disease & 8 & 33.33 & & & 11 & 50.00 & & & 0.397 \\
\hline Pulmonary fibrosis & 4 & 16.67 & & & 8 & 36.36 & & & 0.237 \\
\hline DAS28 & & & 2.51 & $1.13-6.28$ & & & 2.26 & $1.61-5.78$ & 0.757 \\
\hline HAQ-DI & & & 0.38 & $0-2.75$ & & & 1.25 & $0-2.75$ & 0.017 \\
\hline
\end{tabular}

Among poor sleepers, a higher proportion of patients tested positive for rheumatoid factor and anti-citrullinated cyclic peptide (anti$\mathrm{CCP}$ ) autoantibodies. The difference, however, was only statistically significant $(p=0.037)$ for anti-CCP. Although the median DAS28 was comparable in both groups, poor sleepers had a higher frequency of erosive disease $(50.0 \%$ vs $33.3 \%)$ and pulmonary fibrosis (36.4\% vs $16.7 \%)$. Besides, poor sleepers had significantly higher median HAQ-DI $(p=0.017)$ than good sleepers. There was no significant correlation between the global PSQI scores and the following continuous variables: DAS28 $(p=0.589)$, HAQ-DI $(p=0.643)$, and disease duration $(p=0.352)$. Variables, which were significant on univariate analysis (HAQ-DI and anti-C(P), were further analyzed using the logistic regression model. Both HAQ-DI $(p=0.968)$ and anti-CCP $(p=0.431)$ were insignificant when entered in the equation.

\section{DISCUSSION}

Sleep quality is a core component of quality of life which has not received the attention it deserves and hence, not been dealt with adequately in RA. ${ }^{12}$ Our findings demonstrate that patients with RA have significant impairment of sleep quality compared to healthy controls. Previous case-control studies in this regard with healthy controls have reported similar results. ${ }^{3,20}$ Sleep disturbances in RA tend to be multifactorial. Beyond pain, there appears to be a complex and dynamic interplay between the clinical aspects of RA and sleep quality. ${ }^{21}$

This study highlights that functional disability is associated with poor sleep quality. Poor sleepers had significant functional disability (median HAQ-DI of 1.25) as compared to good sleepers who hardly had any difficulty (median HAQ-DI of 0.38). In keeping with our findings, Luyster et al. ${ }^{22}$ and Sariyildiz et al. $^{3}$ found a significant positive correlation between PSQI and HAQ scores. In this study, however, hierarchical regression and multivariate analyses failed to show a significant relationship between HAQ-DI and sleep quality when other variables were entered into the model. The cause-effect relationship between sleep disturbances and functional disability may be bidirectional. This notion was supported by a multi-center, placebo-controlled study which reported that eszopiclone $3 \mathrm{mg}$, a nonbenzodiazepine hypnotic agent, resulted in significant improvement in functional capacity in RA based on the HAQ scores in four weeks of treatment. ${ }^{23}$ Apart from RA, a nexus has been identified between functional limitation and sleep impairment in many other chronic medical conditions. ${ }^{24,25}$

The anti-CCP antibody test is a useful diagnostic and prognostic tool in RA. ${ }^{26}$ Studies have consistently pointed out the role of this antibody as a strong predictor of joint erosions in RA. ${ }^{27}$ RA patients who test positive for anti-CCP antibody are more prone to develop progressive joint damage. ${ }^{28}$ The severity of joint damage tends to parallel the degree of functional 
disability in RA. This may explain our finding of higher HAQ scores and anti-CCP positivity among poor sleepers. To our knowledge, no studies in the literature have reported similar results. Along these lines, Ibn Yacoub et al. ${ }^{29}$ reported that Moroccan RA patients who were anti-CCP positive had higher HAQ scores. Based on our multivariate analyses, neither HAQ-DI nor anti-CCP was an independent predictor of poor sleep quality.

In contrast to previous studies, we detected no link between disease activity and sleep quality. ${ }^{3,30}$ The study by Taylor-Gjevre et al. ${ }^{31}$ demonstrated improved sleep efficiency and "awakening after sleep onset" time observed by polysomnography in RA patients treated with anti-tumor necrosis factor-alpha therapy. However, the mean PSQI scores increased (10.4 to 10.8) with the therapy despite significant reduction in the disease activity $(p=0.048)$. In principle, patients with higher disease activity experience greater pain leading to sleep disturbances. It is not clear why there was no difference in the DAS28 scores between good sleepers and poor sleepers.

We do acknowledge the limitations of our study. Our assessment of sleep quality was based on a questionnaire which is rather subjective. Polysomnography is a more objective alternative in this regard. Furthermore, our small sample size limits the detectable effect size. Besides, a prospective study design can more accurately reveal the relationship between the disease characteristics and sleep quality.

Taken together, the findings of this study demonstrate a link between functional disability, anti-CCP antibodies, and sleep quality in RA. Given the importance of sleep quality, clinicians should routinely perform sleep quality assessments when evaluating patients' response to treatments.

\section{Declaration of conflicting interests}

The authors declared no conflicts of interest with respect to the authorship and/or publication of this article.

\section{Funding}

The authors received no financial support for the research and/or authorship of this article.

\section{REFERENCES}

1. Bai M, Tomenson B, Creed F, Mantis D, Tsifetaki $\mathrm{N}$, Voulgari PV, et al. The role of psychological distress and personality variables in the disablement process in rheumatoid arthritis. Scand J Rheumatol 2009;38:419-30.

2. Escalante A, Del Rincón I. The disablement process in rheumatoid arthritis. Arthritis Rheum 2002;47:333-42.

3. Sariyildiz MA, Batmaz I, Bozkurt M, Bez Y, Cetincakmak MG, Yazmalar L, et al. Sleep quality in rheumatoid arthritis: relationship between the disease severity, depression, functional status and the quality of life. J Clin Med Res 2014;6:44-52.

4. Taylor-Gjevre RM, Gjevre JA, Nair B, Skomro R, Lim HJ. Components of sleep quality and sleep fragmentation in rheumatoid arthritis and osteoarthritis. Musculoskeletal Care 2011;9:152-9.

5. Bollinger T, Bollinger A, Skrum L, Dimitrov S, Lange $\mathrm{T}$, Solbach W. Sleep-dependent activity of T cells and regulatory T cells. Clin Exp Immunol 2009;155:231-8.

6. Cakirbay H, Bilici M, Kavakçi O, Cebi A, Güler M, Tan U. Sleep quality and immune functions in rheumatoid arthritis patients with and without major depression. Int J Neurosci 2004;114:245-56.

7. Besedovsky L, Lange T, Born J. Sleep and immune function. Pflugers Arch 2012;463:121-37.

8. Lange T, Dimitrov S, Born J. Effects of sleep and circadian rhythm on the human immune system. Ann N Y Acad Sci 2010;1193:48-59.

9. Scheff JD, Calvano SE, Lowry SF, Androulakis IP. Modeling the influence of circadian rhythms on the acute inflammatory response. $\mathrm{J}$ Theor Biol 2010;264:1068-76.

10. Dimitrov S, Lange T, Tieken S, Fehm HL, Born J. Sleep associated regulation of $\mathrm{T}$ helper $1 / \mathrm{T}$ helper 2 cytokine balance in humans. Brain Behav Immun 2004; 18:341-8.

11. Løppenthin K, Esbensen BA, Jennum P, Østergaard M, Christensen JF, Thomsen T, et al. Effect of intermittent aerobic exercise on sleep quality and sleep disturbances in patients with rheumatoid arthritis - design of a randomized controlled trial. BMC Musculoskelet Disord 2014;15:49.

12. Wells $\mathrm{G}, \mathrm{Li} \mathrm{T}$, Tugwell $\mathrm{P}$. Investigation into the impact of abatacept on sleep quality in patients with rheumatoid arthritis, and the validity of the MOSSleep questionnaire Sleep Disturbance Scale. Ann Rheum Dis 2010;69:1768-73.

13. Aletaha D, Neogi T, Silman AJ, Funovits J, Felson DT, Bingham CO, et al. 2010 rheumatoid arthritis classification criteria: an American College of Rheumatology/European League Against Rheumatism collaborative initiative. Ann Rheum Dis 2010;69:1580-8.

14. Fransen J, van Riel PL. The Disease Activity Score and the EULAR response criteria. Clin Exp Rheumatol 2005;23:93-9. 
15. Prevoo ML, van 't Hof MA, Kuper $\mathrm{HH}$, van Leeuwen MA, van de Putte LB, van Riel PL. Modified disease activity scores that include twenty-eight-joint counts. Development and validation in a prospective longitudinal study of patients with rheumatoid arthritis. Arthritis Rheum 1995;38:44-8.

16. Mäkinen $H$, Kautiainen $H$, Hannonen $P$, Sokka T. Is DAS28 an appropriate tool to assess remission in rheumatoid arthritis? Ann Rheum Dis 2005;64:1410-3.

17. Maska L, Anderson J, Michaud K. Measures of functional status and quality of life in rheumatoid arthritis: Health Assessment Questionnaire Disability Index (HAQ), Modified Health Assessment Questionnaire (MHAQ), Multidimensional Health Assessment Questionnaire (MDHAQ), Health Assessment Questionnaire II (HAQ-II), Improved Health Assessment Questionnaire (Improved HAQ), and Rheumatoid Arthritis Quality of Life (RAQoL). Arthritis Care Res (Hoboken) 2011;63:4-13.

18. Bruce B, Fries JF. The Stanford Health Assessment Questionnaire: dimensions and practical applications. Health Qual Life Outcomes 2003;1:20.

19. Buysse DJ, Reynolds CF, Monk TH, Berman SR, Kupfer DJ. The Pittsburgh Sleep Quality Index: a new instrument for psychiatric practice and research. Psychiatry Res 1989;28:193-213.

20. Son CN, Choi G, Lee SY, Lee JM, Lee TH, Jeong $\mathrm{HJ}$, et al. Sleep quality in rheumatoid arthritis, and its association with disease activity in a Korean population. Korean J Intern Med 2015;30:384-90.

21. Drewes AM, Svendsen L, Taagholt SJ, Bjerregård K, Nielsen KD, Hansen B. Sleep in rheumatoid arthritis: a comparison with healthy subjects and studies of sleep/wake interactions. Br J Rheumatol 1998;37:71-81.

22. Luyster FS, Chasens ER, Wasko MC, Dunbar-Jacob J. Sleep quality and functional disability in patients with rheumatoid arthritis. J Clin Sleep Med 2011;7:49-55.

23. Roth T, Price JM, Amato DA, Rubens RP, Roach JM, Schnitzer TJ. The effect of eszopiclone in patients with insomnia and coexisting rheumatoid arthritis: a pilot study. Prim Care Companion J Clin Psychiatry 2009;11:292-301.

24. Kim J, Kim Y, Yang KI, Kim DE, Kim SA. The Relationship Between Sleep Disturbance and Functional Status in Mild Stroke Patients. Ann Rehabil Med 2015;39:545-52.

25. Schurman JV, Friesen CA, Dai H, Danda CE, Hyman PE, Cocjin JT. Sleep problems and functional disability in children with functional gastrointestinal disorders: an examination of the potential mediating effects of physical and emotional symptoms. BMC Gastroenterol 2012;12:142.

26. Niewold TB, Harrison MJ, Paget SA. Anti-CCP antibody testing as a diagnostic and prognostic tool in rheumatoid arthritis. QJM 2007;100:193-201.

27. Jilani AA, Mackworth-Young CG. The role of citrullinated protein antibodies in predicting erosive disease in rheumatoid arthritis: a systematic literature review and meta-analysis. Int $\mathrm{J}$ Rheumatol 2015;2015:728610.

28. de Vries-Bouwstra JK, Goekoop-Ruiterman YP, Verpoort KN, Schreuder GM, Ewals JA, Terwiel JP, et al. Progression of joint damage in early rheumatoid arthritis: association with HLA-DRB1, rheumatoid factor, and anti-citrullinated protein antibodies in relation to different treatment strategies. Arthritis Rheum 2008;58:1293-8.

29. Ibn Yacoub Y, Amine B, Laatiris A, Hajjaj-Hassouni N. Rheumatoid factor and antibodies against citrullinated peptides in Moroccan patients with rheumatoid arthritis: association with disease parameters and quality of life. Clin Rheumatol 2012;31:329-34.

30. Houssien DA, McKenna SP, Scott DL. The Nottingham Health Profile as a measure of disease activity and outcome in rheumatoid arthritis. $\mathrm{Br} \mathrm{J}$ Rheumatol 1997;36:69-73.

31. Taylor-Gjevre RM, Gjevre JA, Nair BV, Skomro RP, Lim HJ. Improved Sleep Efficiency after Anti-Tumor Necrosis Factor $\alpha$ Therapy in Rheumatoid Arthritis Patients. Ther Adv Musculoskelet Dis 2011;3:227-33. 